\title{
]jfis
}

\section{RLC Fault Detection Based on Image Processing and Artificial Neural Network}

Ali Rohan ${ }^{1}$ and Sung Ho Kim ${ }^{2}$

${ }^{1}$ School of Electronics and Information Engineering, Kunsan National University, Kunsan, Korea

${ }^{2}$ School of IT, Information and Control Engineering, Kunsan National University, Kunsan, Korea

\begin{abstract}
The RLC circuit is a basic building block of the more complicated electrical circuits and networks. RLC circuit mainly comprises of passive electronic components such as resistances, capacitors, and inductors. When used in an electrical circuit, these electronic components, lose their ability to perform properly due to continuous use and an aging factor. In some cases, these components confront with short circuit and get damaged due to high current. These damaged components get faulty. In the case of prolonging and continuous use, these faulty components, disturb the performance of the electrical circuit and in case of short circuit, totally stops the electrical circuit from performing some specific tasks. In this work, a fault diagnosis system for the detection of these faulty RLC electronic components is proposed. The proposed fault diagnosis system is based on a fault detection scheme comprising of image processing and artificial neural network. The proposed system is designed in MATLAB/Simulink. The system is tested under different fault scenarios and performance is evaluated. The simulation results have proved that the proposed system can diagnose the faulty electronic components effectively, within and outside an electrical circuit.
\end{abstract}

Keywords: Feature extraction, Fault diagnosis, RLC faults, Image processing, Artificial neural network

\section{Introduction}

An electrical circuit is mainly composed of electronic components, combined together to perform a specific task in an electrical system. These electronic components are mainly divided into two categories: active electronic components and passive electronic components. Passive

Received: Sep. 13, 2018

Revised : Jun. 9, 2019

Accepted: Jun. 10, 2019

Correspondence to: Ali Rohan (ali_rohan2003@hotmail.com)

(CThe Korean Institute of Intelligent Systems

cCThis is an Open Access article distributed under the terms of the Creative Commons Attribution Non-Commercial License (http://creativecommons.org/licenses/ by-nc/3.0// which permits unrestricted noncommercial use, distribution, and reproduction in any medium, provided the original work is properly cited. electronic components comprise of resistance (R), inductor (L) and capacitor (C), commonly combined to form an RLC circuit. The RLC circuits are basically used to make variable tuned circuits, filters, oscillators, voltage multipliers, amplifiers, and pulse discharge circuits. The RLC electronic components are made up of some specific materials with different power ratings. Each component is made to hold a limited amount of current and voltage. In case of capacitors and inductors, components are designed to store a limited amount of electric and magnetic charge. As time goes by, these electronic components used in an electrical circuit, lose their ability to perform up to their designed rating due to prolong and continuous use and an aging factor. For electronic components, there are basically two general aging progressive failure modes (degraded performance, functional failures) and two end state aging failure modes (short circuit, open circuit) [1--3]. 
Progressive failures occur when an electronic component is old or used for a long time in a circuit. The effect of the progressive failures are degradation in the performance of the component and unable to perform some specific functions. Whereas end state failures occur when a component is exposed to very high electrical current and in this case, the electronic component totally stops working.

Previously, different methods to diagnose electronic components faults and failures has been proposed. In [4, 5], the authors used reliability modeling; a method to analyze circuit reliability, using accelerated failure data, and quantifying the reliability of multiple components on a circuit board. In [6], the authors proposed a signal comparison technique; a comparison process to identify deviations from the normal signal within the circuit board. For the fault diagnosis and identification systems, in [7], a DWT and artificial neural network-based fault detection and identification system is proposed. Whereas, in [8-10], current signature analysis is used for the detection and identification of faults. In [11], a fault diagnosis and monitoring system using Kohonen Neural Network (KNN) was proposed. An online diagnosis method using flux signals was described in [12]. A method based on feed-forward multilayer neural network was presented in [13]. Fuzzy logic-based fault detection system was developed for implementation on emergency vehicles in [14]. In [15] authors evaluated wavelet-based schemes based on neural network and support vector machine (SVM) for fault detection and classification. An artificial neural networkbased fault diagnosis system using feedforward neural network was presented [16].

These fault diagnosis systems normally comprise of one strategy for detection, only a neural network is used for the detection of a fault. Every time a fault is diagnosed the system goes through complex calculations and feature extraction process. Whereas, in this work, a fault diagnosis system based on a fault detection scheme comprising of image processing and the artificial neural network is proposed. The system is divided into two strategies rather than only one strategy. Fault segregation based on fault intensity is performed to judge if the fault requires to pass through complex calculations and feature extraction process. Image processing is used as a first strategy and it performs coarse diagnosis for fault detection. If the fault is easy to distinguish, no more processing will be performed but if it's hard to diagnose the fault then second strategy based on artificial neural network performs fine diagnosis for fault detection. First, a database is formed using the normal passive electronic components. The database consists of voltage and current values and VI response of the components. To test a component that either it is faulty or not, a constant voltage is applied across the electronic component within or outside an electrical circuit, voltage and current response is recorded and VI curve is plotted. The plotted VI curve is converted into an image and is compared with the image formed from already saved database values of the electronic component of same rating and type. If the difference between the image is big from a specifically defined value, the artificial neural network is activated to double check the condition that either the component is faulty or not and calculates the fault probability. The proposed system is designed in MATLAB/Simulink. The system is tested under different fault scenarios and performance is evaluated. The simulation results have proved that the proposed system can diagnose the faulty electronic components effectively, within and outside an electrical circuit.

This paper is divided into four sections. Section 2 describes the structure of fault diagnosis system. Section 3 is composed of simulation studies and results are discussed. Section 4 outlines the conclusion.

\section{Structure of Proposed Fault Diagnosis Sys- tem}

The proposed fault diagnosis system is divided into three parts:

1) Database formation.

2) Coarse diagnosis.

3) Fine diagnosis.

\subsection{Database Formation}

The first step of the proposed fault diagnosis system is to make a database from normal passive electronic components. The database is required for the comparison between the normal and faulty electronic components. Figure 1 shows the block diagram to make a database.

The constant voltage across the normal RLC component is applied and voltage and current across the components are measured. VI curve is plotted from the measured voltage and current values. Image processing is used to convert the plotted curve into an image format and is stored in the database along with the extracted features. 


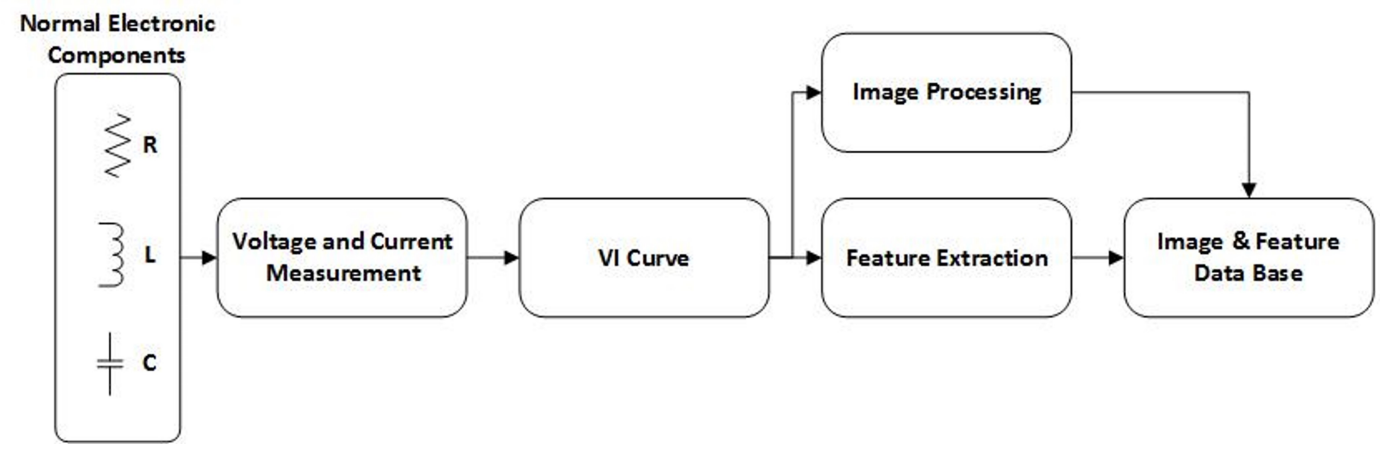

Figure 1. Block diagram for making database.

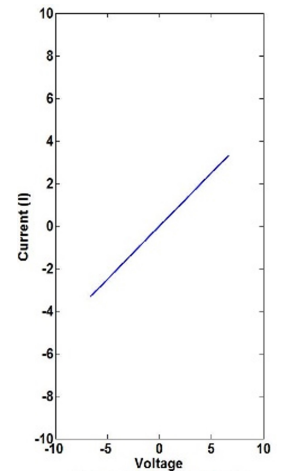

(a)

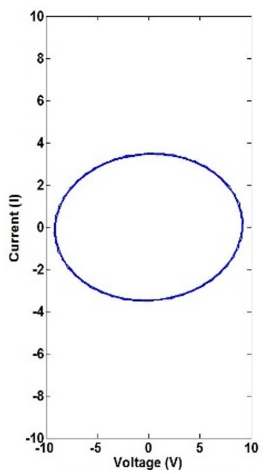

(b)

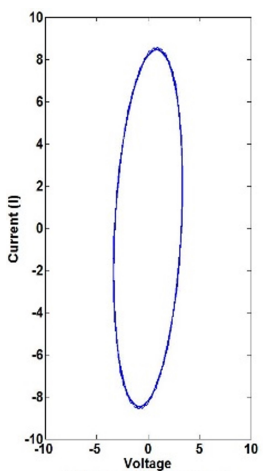

(c)

Figure 2. VI curve for (a) resistance, (b) capacitor, and (c) inductor.

\subsection{Coarse Diagnosis}

Figure 2 shows the VI curves of the normal R (resistance), L (inductor) and $\mathrm{C}$ (capacitor) components. It can be observed that in the case of R, the VI curve is just a linear straight line. Whereas, in the case of $\mathrm{L}$ and $\mathrm{C}$, the VI curve is the shape of an ellipse. Therefore, for the diagnosis of faulty R components, only the slope is calculated and compared with stored normal component slope value but for $\mathrm{L}$ and $\mathrm{C}$ components, an additional diagnostic algorithm is required. For that, image processing and the artificial neural network is used.

In order to test an electronic component, coarse diagnosis is done to check the fault proportion. If the proportion is big, fine diagnosis is performed to further diagnose the fault. Figure 3 shows the block diagram of coarse and fine diagnosis. In coarse diagnosis, the constant voltage across the electronic component is applied and the VI curve is plotted from recorded voltage and current values. The plotted VI curve is converted into an image and is compared with the already stored image of the normal component. Image pattern matching algorithm is applied to compare both images and the correlation coefficient

is calculated using Eq. (1).

$$
\rho=\frac{\sum_{m} \sum_{n}\left(A_{m n}-\bar{A}\right)\left(B_{m n}-\bar{B}\right)}{\sqrt{\left(\sum_{m} \sum_{n}\left(A_{m n}-\bar{A}\right)^{2}\right)}\left(\sum_{m} \sum_{n}\left(B_{m n}-\bar{B}\right)^{2}\right)},
$$

where

$\rho=$ Correlation coefficient,

$A=$ Matrix containing normal component image pixel values,

$B=$ Matrix containing faulty component image pixel values,

$m=$ Number of rows,

$n=$ Number of columns.

The calculated value of correlation coefficient is compared with a threshold value, if the value of correlation coefficient is greater than a threshold value, it indicates that the tested component is faulty and activates the fine diagnosis to diagnose the fault more precisely.

\subsection{Fine Diagnosis}

In fine diagnosis, suitable features are extracted from the VI curve of the component underdiagnosis. After extracting suitable features, a feature vector is made depending on the type of the component i.e., inductor or capacitor.

Figure 3 shows the block diagram of the fine diagnosis. As mentioned before, for $\mathrm{R}$ only slope is enough to determine the faulty component. For $\mathrm{L}$ and $\mathrm{C}$, the extracted features are shown in Table 1.

\subsubsection{Artificial neural network}

The architecture of the neural network used in this fault diagnosis method is a feed-forward network. With the six inputs, neural network is composed of one input layer consists of six 


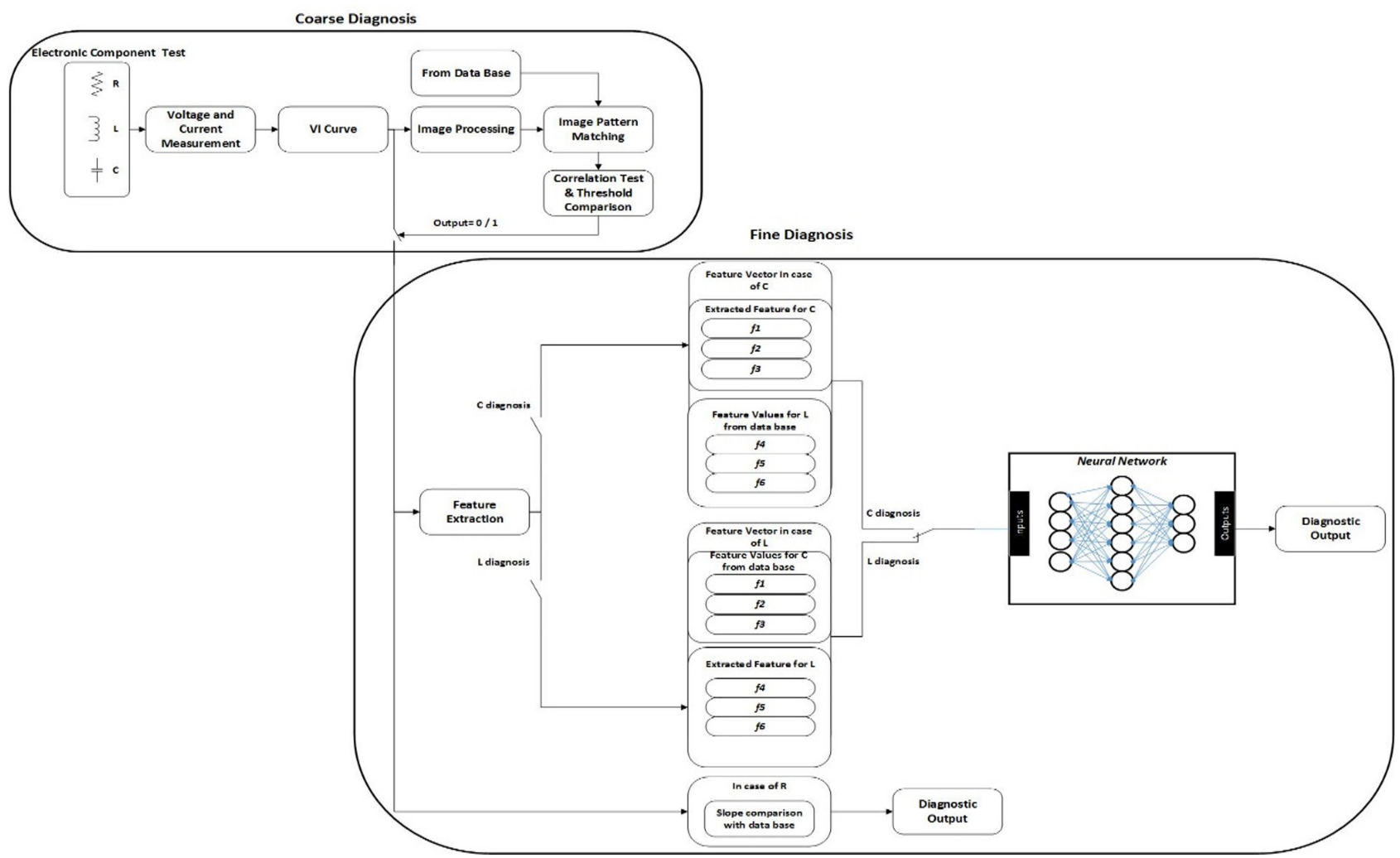

Figure 3. Block diagram of the fault diagnosis system.

Table 1. Extracted features names and description

\begin{tabular}{ccl}
\hline $\begin{array}{c}\text { Feature } \\
\text { value }\end{array}$ & Feature name & Feature description \\
\hline$f 1$ & Eccentricity C & $\begin{array}{l}\text { Eccentricity of the VI curve } \\
\text { for capacitor }\end{array}$ \\
$f 2$ & Elliptical area C & $\begin{array}{l}\text { Area of the ellipse in VI curve for } \\
\text { capacitor }\end{array}$ \\
$f 3$ & $\begin{array}{c}\text { Eccentric angle } \\
\text { Eccentric angle of the ellipse in } \\
\text { VI curve for capacitor }\end{array}$ \\
$f 4$ & Eccentricity L & $\begin{array}{l}\text { Eccentricity of the VI curve } \\
\text { for inductor }\end{array}$ \\
$f 5$ & $\begin{array}{c}\text { Elliptical area } \\
\text { Area of the ellipse in VI curve } \\
\text { for inductor }\end{array}$ \\
\hline$f 6$ & $\begin{array}{c}\text { Eccentric angle } \\
\text { L }\end{array}$ & $\begin{array}{l}\text { Eccentric angle of the ellipse } \\
\text { in VI curve for inductor }\end{array}$ \\
\hline
\end{tabular}

neurons, one hidden layer with 20 neurons and one output layer with two neurons and target output of 0 or 1 , each of which represents different health condition of the system. The sigmoid activation function is used for hidden and output layers. In the start, the neural network is usually trained with the dataset of normal and faulty data. After that, trained network is further used for fault detection and diagnosis purpose.

A feed-forward artificial neural network is used to determine the probability of fault. A single neural network is used and trained for both inductor and capacitor cases. In case of a capacitor, $\mathrm{C}$ diagnosis switches are turned on and $\mathrm{L}$ diagnosis switches are turned off. Features are extracted from the VI curve of the component and feature vector comprising of six feature values $f 1, f 2, \ldots, f 6$ is made. $f 1, f 2, f 3$ are the feature values of the extracted features for capacitor underdiagnosis and $f 4, f 5, f 6$ are the feature values of the inductor from the database. Whereas, in the case of an inductor, L diagnosis switches are turned on and $\mathrm{C}$ diagnosis switches are turned off. Features are extracted from the VI curve. In this case, $f 4, f 5, f 6$ are the feature values of the extracted features for inductor underdiagnosis and $f 1, f 2, f 3$ are the feature values of capacitor from the database. The diagnostic output of the neural network varies from 0 to 1 from normal to probability of fault respectively.

The features are extracted using basic ellipse equations for capacitor and inductor. For resistance, the only slope is calculated. Equations used are given as:

$$
\begin{aligned}
& \text { Eccentricity }=\sqrt{1-\frac{b^{2}}{a^{2}}}, \\
& \text { Elliptical area }=\pi a b, \\
& \text { Eccentric angle }=\tan ^{-1}\left(\frac{b}{a}\right),
\end{aligned}
$$




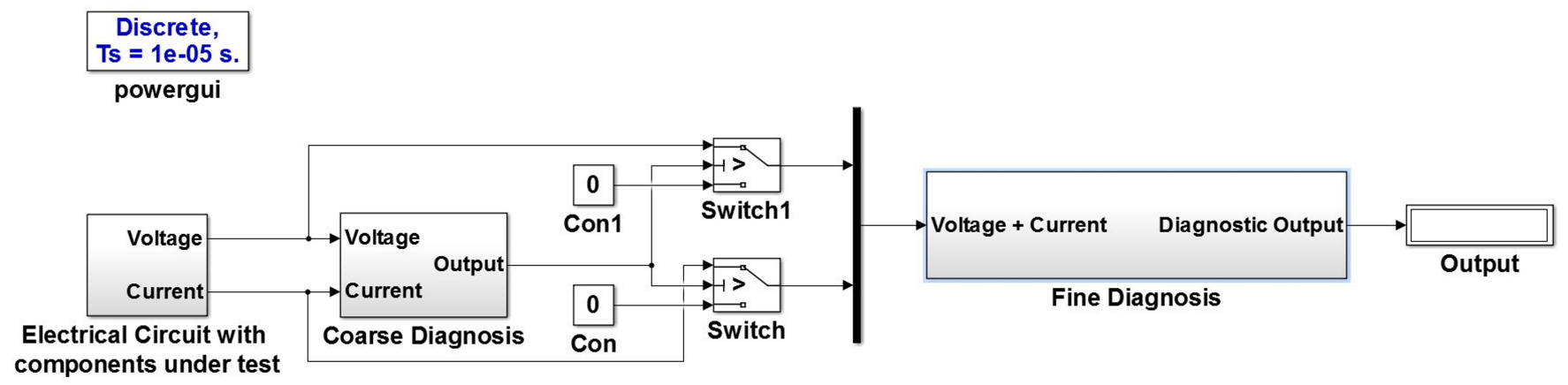

Figure 4. Basic Simulink model for fault diagnosis system.

$$
\text { Slope for } \mathrm{R}=\frac{y_{2}-y_{1}}{x_{2}-x_{1}},
$$

where

$b=$ Semi-minor axis of the ellipse,

$a=$ Semi-major axis of the ellipse,

$y_{2}-y_{1}=$ Difference of maximum and minimum value of the $y$-coordinate,

$x_{2}-x_{1}=$ Difference of maximum and minimum value of the $x$-coordinate.

\section{Simulation Studies}

The proposed fault diagnosis system was modeled in MATLAB/Simulink. The Simulink model of the system is shown in Figure 4.

Figure 4 shows a basic Simulink model for the fault diagnosis system. The Simulink model consists of three main subsystems: electrical circuit with the component under test, coarse diagnosis, and fine diagnosis.

The first subsystem is a basic electrical circuit which is composed of resistors, inductors, and capacitors. Figure 5 shows the designed electrical circuit for the component test. It can be seen in Figure 5 that a probe for testing the electronic equipment is used. This probe is basically to provide some voltage across the component under test. Voltage is applied to record the VI response of the component. In this case, the applied voltage is $10 \mathrm{~V}$. Figure 6 shows the probe designed for testing of the electronic component.

The second subsystem in Figure 4 is the coarse diagnosis. As mentioned previously, coarse diagnosis is done to get the initial state of the electronic component that how healthy a component is using image processing and image pattern matching algorithm. Figure 7 shows the coarse diagnosis subsystem. It can be seen in Figure 7 that voltage and current of the compo- nent under test with normal VI data are given as an input to the function which computes and implies the image processing algorithm and calculates the correlation between the normal VI and tested VI image. If the correlation value is less than a threshold value, which in this case is 0.6 , the switch of the fine diagnosis is turned on and further diagnosis is performed using the artificial neural network.

The third subsystem in Figure 4 is the fine diagnosis. When the correlation value is less than 0.6 , fine diagnosis is activated. In fine diagnosis, voltage and current value from the electronic component under test go through a feature extraction process. Features are extracted and output is given to a neural network in the form of a feature vector containing 6 values. If the device under test is an inductor, the feature vector given at the input of the neural network contains 6 feature values $f 1, f 2, \ldots, f 6$. First three feature values $f 1, f 2, f 3$ are related to a capacitor which in this case are chosen from the database and normal feature values are given. Last three features $f 4, f 5, f 6$ are the feature values extracted in real time from the faulty inductor component under test. Whereas, if the device under test is a capacitor, the feature vector contains 6 feature values but, in this case, $f 1, f 2, f 3$ are the features values extracted in real time from the faulty capacitor. The output of neural network changes from 0 to 1 i.e., normal to faulty. Figure 8 shows the fine diagnosis Simulink model.

\subsection{Results}

Several simulations were carried out to test the proposed fault diagnosis system using inductors, capacitors, and resistances of different values. One of the simulation results is presented for an inductor of the inductance of $1 e^{-3}$ and a capacitor of capacitance $1 e^{-3}$.

For a capacitor of capacitance $1 e^{-3}$, the VI curve of the normal component is shown in Figure 9. It can be seen that 


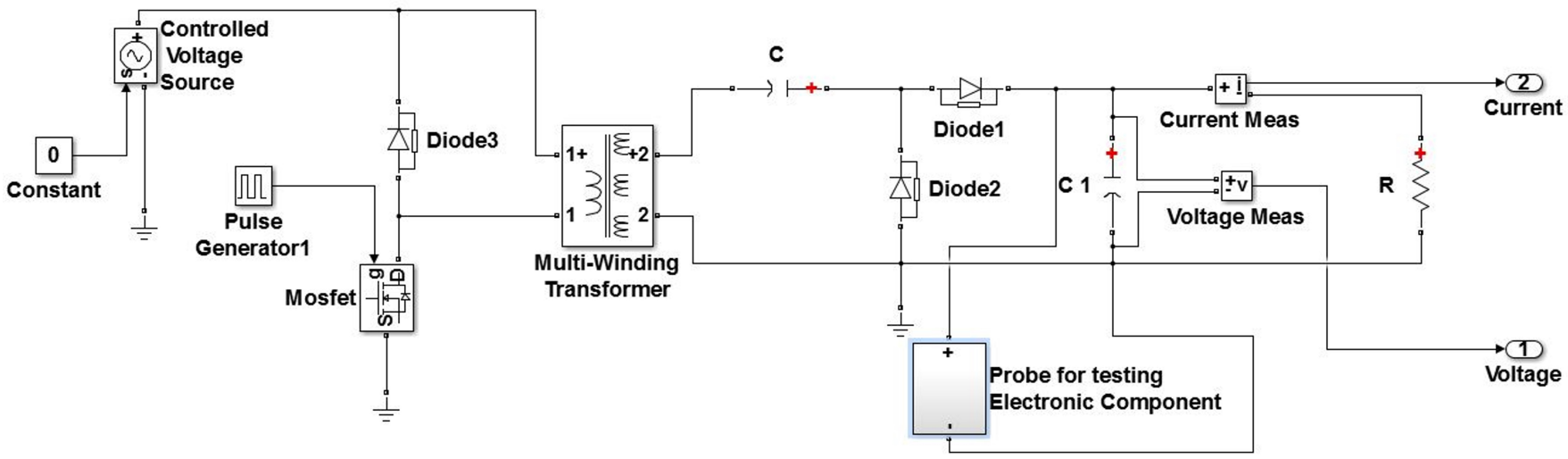

Figure 5. Designed electrical circuit with electronic component under test.

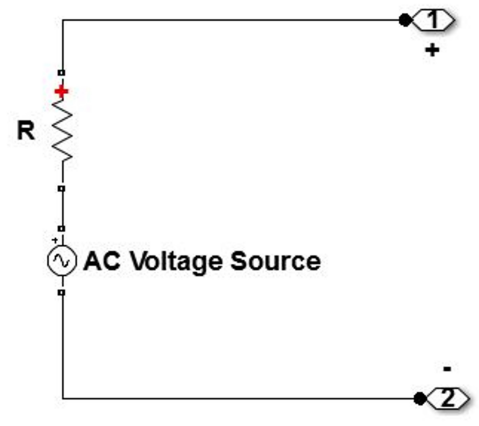

Figure 6. Probe for electronic component testing.

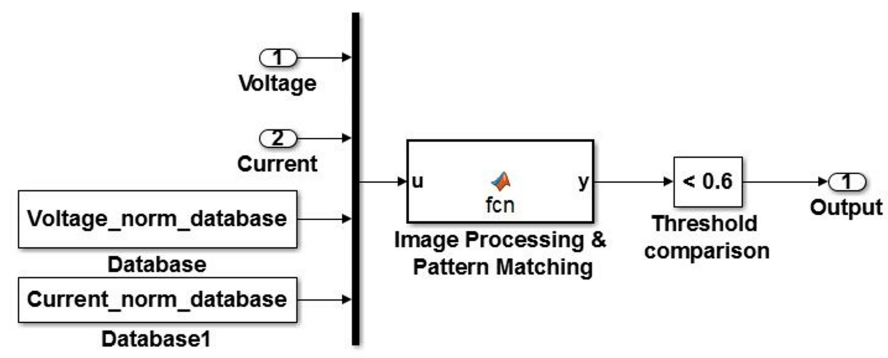

Figure 7. Coarse diagnosis subsystem.

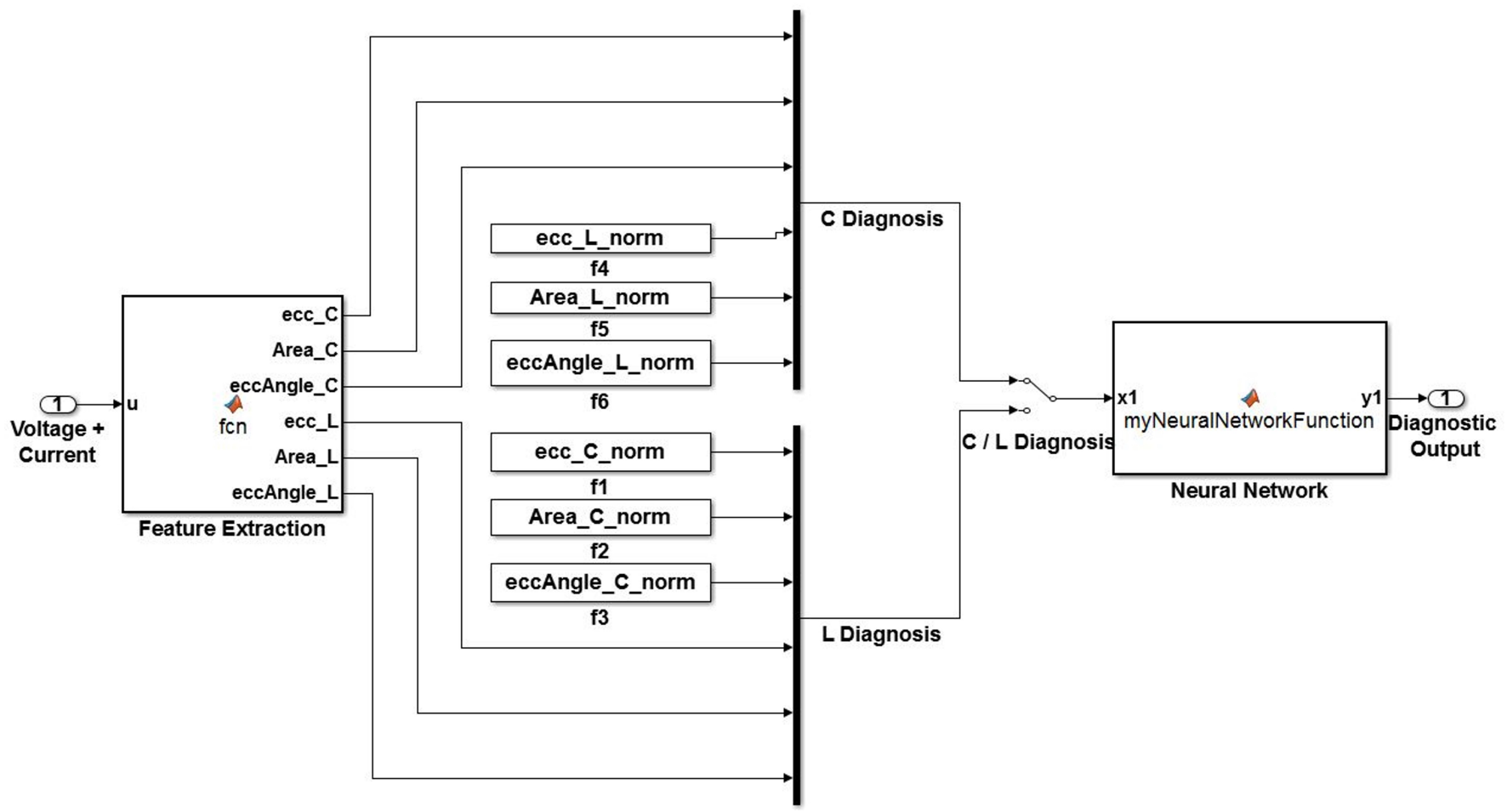

Figure 8. Fine diagnosis subsystem. 


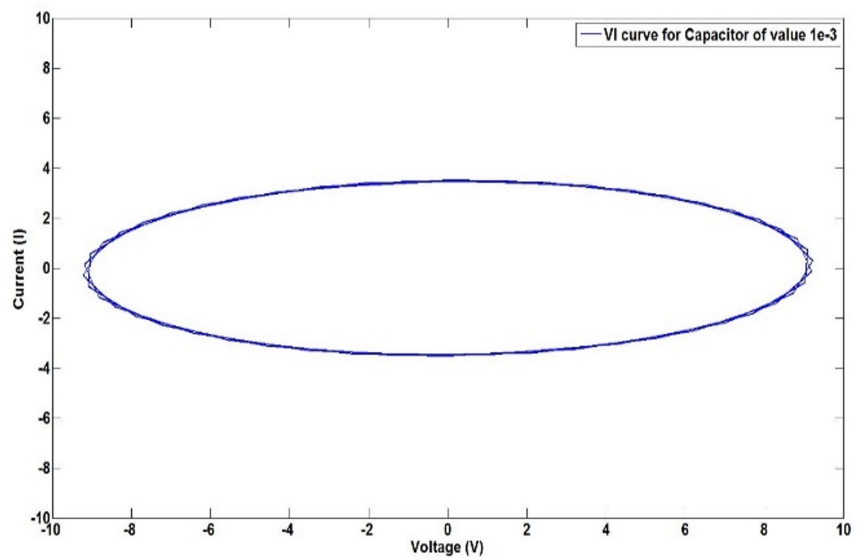

Figure 9. VI curve for a normal capacitor of value $1 e^{-3}$.

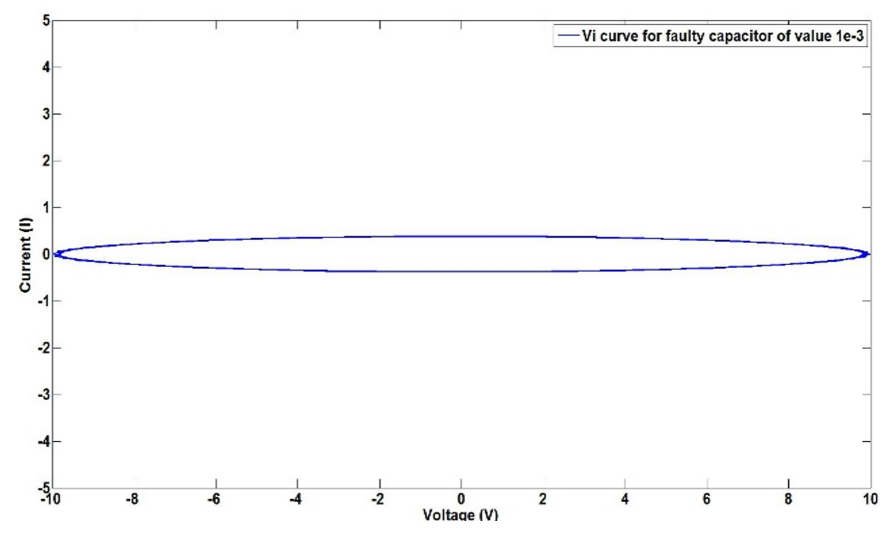

Figure 10. VI curve for a faulty capacitor of value $1 e^{-3}$.

the VI curve of a capacitor having capacitance $1 e^{-3}$ is ellipse parallel to the $\mathrm{x}$-axis. In the Simulink model, the fault is inserted by changing the capacitance from $1 e^{-3}$ to $1 e^{-4}$, Figure 10 shows the VI curve of the faulty capacitor.

For an inductor of inductance $1 e^{-3}$, the VI curve for the normal component is shown in Figure 11 and for the faulty inductor, the inductance value is changed from $1 e^{-3}$ to $1 e^{-4}$. VI curve for a faulty inductor is shown in Figure 12.

After inserting the fault in the capacitor and inductor values, the diagnostic algorithm is activated and first, the correlation was checked using image processing, which in this case was less than 0.6 (set threshold value), the fine diagnosis was done by extracting features from VI response of the components. Figure 13 shows the $\mathrm{C}$ diagnosis results for a normal capacitor of capacitance $1 e^{-3}$. It can be seen in the Figure 13 that because the component is normal without any fault, the first output of neural network for $\mathrm{C}$ is almost 0 and the second output is also 0 because the diagnosis is $\mathrm{C}$ diagnosis and component under test is a capacitor. Figure 14 shows the $\mathrm{C}$ diagnosis result for a

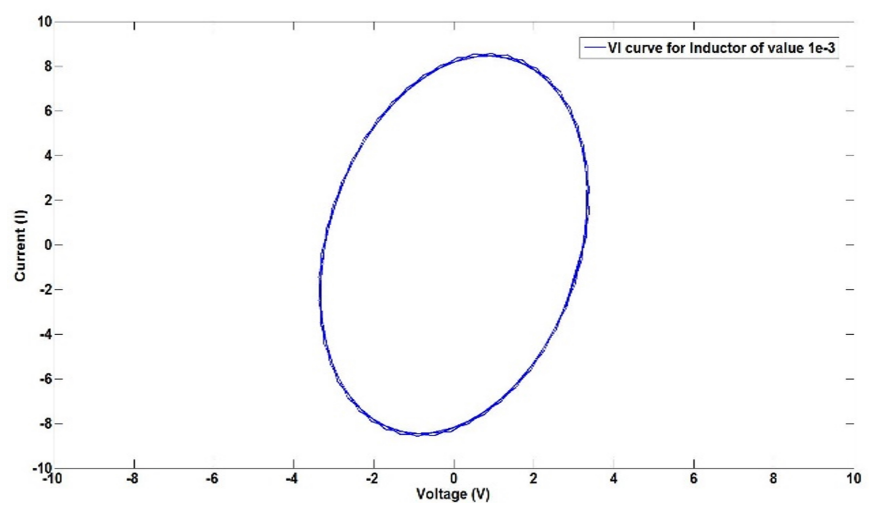

Figure 11. VI curve for the normal inductor of value $1 e^{-3}$.

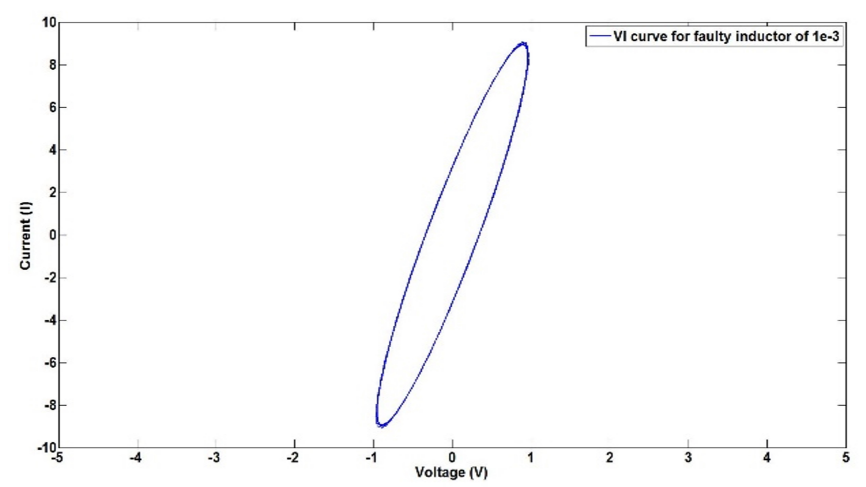

Figure 12 . VI curve for the faulty inductor of value $1 e^{-3}$.

faulty capacitor of capacitance $1 e^{-3}$. The fault was inserted by changing the capacitance value of the normal capacitor, neural network output is 1 , which indicates that the component is faulty. Figure 15 shows the L diagnosis result for a normal inductor of inductance $1 e^{-3}$. Figure 16 shows the result for a faulty inductor of inductance $1 e^{-3}$.

The system was tested for different set of values of capacitors and inductor. Simulations were carried out by changing the capacitor and inductor of different values. Artificial neural network performed well to diagnose the faulty component. The accuracy achieved was $98.87 \%$ (calculated for several fault scenarios). The output results show that the proposed fault diagnosis system works well to diagnose the faults caused by progressive and end state failure modes.

\section{Conclusion}

An effective and efficient method to diagnose the faulty RLC component in an electrical circuit is proposed in this work. MATLAB/Simulink is used to model and implement the proposed method. The system works on fault diagnosis scheme 


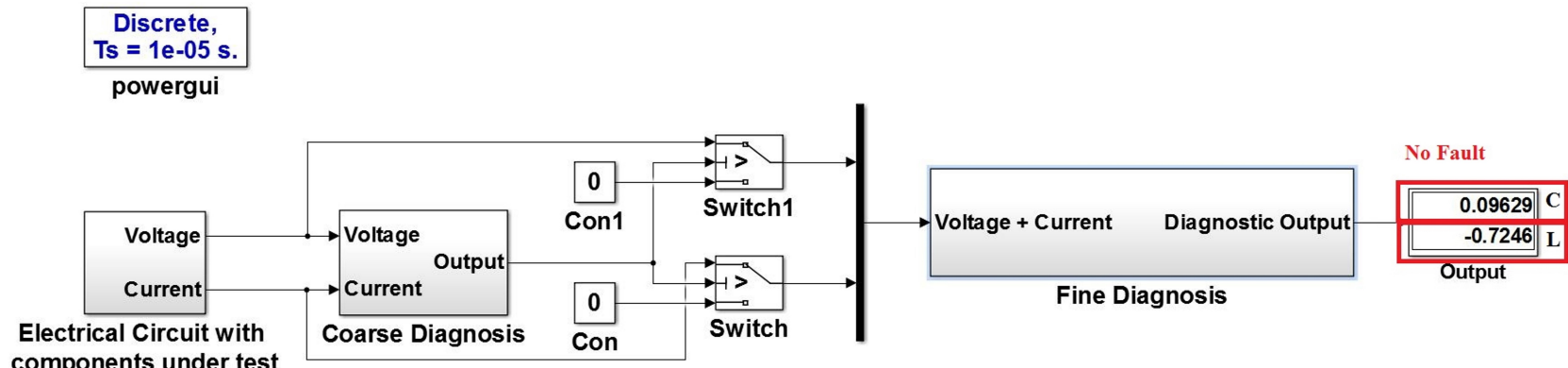

Figure 13. C diagnosis results for normal capacitor of capacitance $1 e^{-3}$.

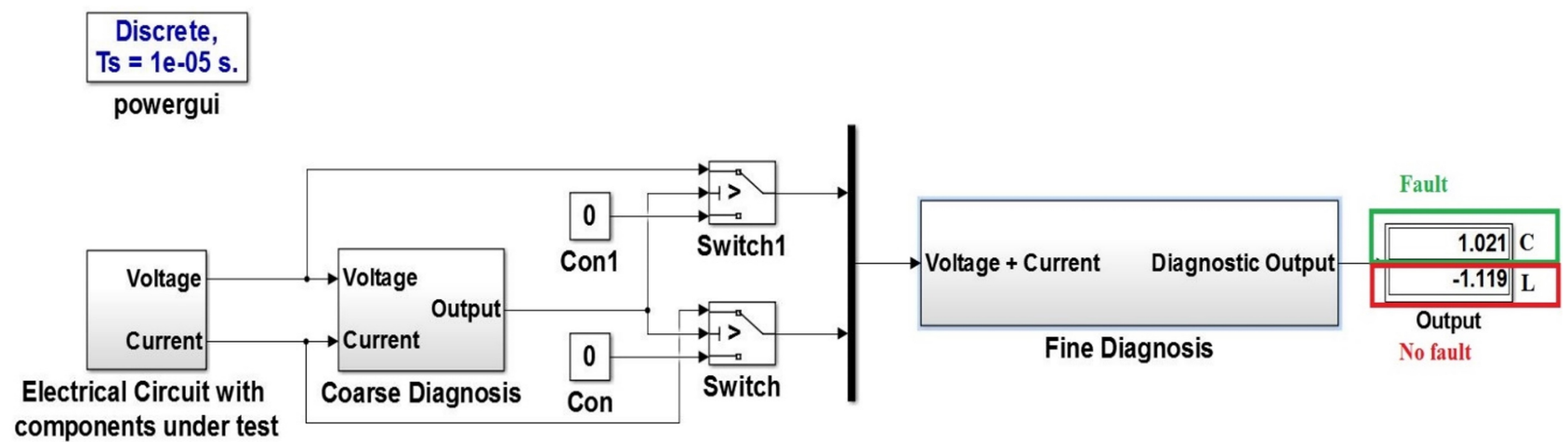

Figure 14. C diagnosis result for a faulty capacitor of capacitance $1 e^{-3}$.

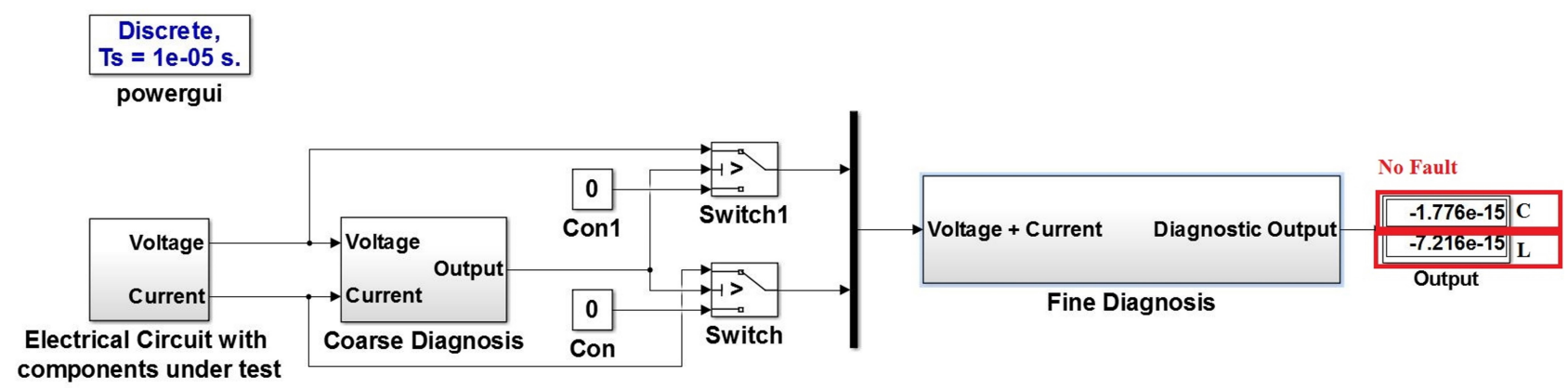

Figure 15. L diagnosis result for a normal inductor of inductance $1 e^{-3}$.

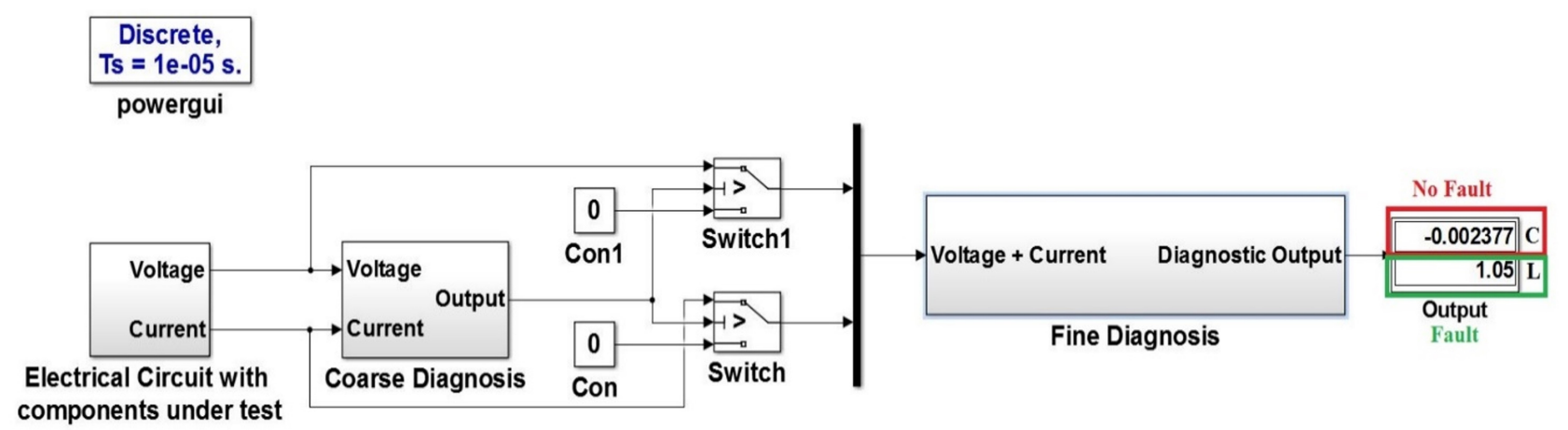

Figure 16. L diagnosis result for a faulty inductor of inductance $1 e^{-3}$. 
based on image processing and artificial neural network. The system is tested for several electronic components of different values. The simulation results show that the proposed fault diagnosis system is able enough to diagnose the faults for RLC component in an electrical circuit.

\section{Conflict of Interest}

No potential conflict of interest relevant to this article was reported.

\section{References}

[1] Electronic Power Research Institute, "Collected field data on electronic part failures and aging in nuclear power plant instrumentation and control (I\&C) systems," Electronic Power Research Institute, Palo Alto, CA, Technical Report 1003568, 2002.

[2] Electronic Power Research Institute, "Printed circuit board maintenance, repair, and testing guide," Electronic Power Research Institute, Palo Alto, CA, Technical Report 1007916, 2003.

[3] Electronic Power Research Institute, "Guidelines for the monitoring of aging of I\&C electronic components," Electronic Power Research Institute, Palo Alto, CA, Technical Report 1008166, 2004.

[4] S. D. Bhakta, S. Lundberg, and G. Mortensen, "Accelerated tests to simulate metal migration in hybrid circuits," in Proceedings of Annual Reliability and Maintainability Symposium (Cat. No. 02CH37318), Seattle, WA, 2002, pp. 319-324. http://doi.org/10.1109/RAMS.2002.981661

[5] R. Valentin, M. Osterman, and B. Newman, "Remaining life assessment of aging electronics in avionic applications," in Proceedings of Annual Reliability and Maintainability Symposium, Tampa, FL, 2003, pp. 313-318. http://doi.org/10.1109/RAMS.2003.1182008

[6] H. Kim, H. J. Jeon, K. Lee, and H. Lee, "The design and evaluation of all voting triple modular redundancy system," in Proceedings of Annual Reliability and Maintainability Symposium (Cat. No. 02CH37318), Seattle, WA, 2002, pp. 439-444. http://doi.org/10.1109/RAMS.2002.981682

[7] A. Rohan and S. H. Kim, "Fault detection and diagnosis system for a three-phase inverter using a DWT-based artificial neural network," International Journal of Fuzzy
Logic and Intelligent Systems, vol. 16, no. 4, pp. 238-245, 2016. https://doi.org/10.5391/IJFIS.2016.16.4.238

[8] A. Rohan, M. Rabah, and S. H. Kim, "An integrated fault detection and identification system for permanent magnet synchronous motor in electric vehicles," International Journal of Fuzzy Logic and Intelligent Systems, vol. 18, no. 1, pp. 20-28, 2018. https://doi.org/10.5391/IJFIS.2018. 18.1 .20

[9] M. Talha, F. Asghar, and S. H. Kim, "A Matlab and Simulink based three-phase inverter fault diagnosis method using three-dimensional features," International Journal of Fuzzy Logic and Intelligent Systems, vol. 16, no. 3, pp. 173-180, 2016. https://doi.org/10.5391/IJFIS. 2016.16.3.173

[10] F. Asghar, M. Talha, and S. H. Kim, "Comparative study of three fault diagnostic methods for three phase inverter with induction motor," International Journal of Fuzzy Logic and Intelligent Systems, vol. 17, no. 4, pp. 245-256, 2017. https://doi.org/10.5391/IJFIS.2017.17.4.245

[11] B. S. Yang, T. Han, and J. L. An, "ART-KOHONEN neural network for fault diagnosis of rotating machinery," Mechanical Systems and Signal Processing, vol. 18, no. 3, pp. 645-657, 2004. https://doi.org/10.1016/S08883270(03)00073-6

[12] M. Bouzid, G. Champenois, N. Bellaaj, and K. Jelassi, "Automatic and robust diagnosis of broken rotor bars fault in induction motor," in Proceedings of the 19th International Conference on Electrical Machines (ICEM), Rome, Italy, 2010, pp. 1-7. https://doi.org/10.1109/ICELMACH. 2010.5608108

[13] R. Bayir and O. F. Bay, "Serial wound starter motor faults diagnosis using artificial neural network," in Proceedings of the IEEE International Conference on Mechatronics (ICM), Istanbul, Turkey, 2004, pp. 194-199. https://doi. org/10.1109/ICMECH.2004.1364436

[14] R. Bayir and O. Bay, "A fault diagnosis of engine starting system via starter motors using fuzzy logic algorithm," Gazi University Journal of Science, vol. 24, no. 3, pp. 437-449, 2011.

[15] S. G. Vijay, S. H. Kumar, P. P. Srinivasa, S. N. Sriram, and R. B. Rao, "Evaluation of effectiveness of wavelet based denoising schemes using ANN and SVM 
for bearing condition classification," Computational Intelligence and Neuroscience, vol. 2012, article no. 16, 2012. https://doi.org/10.1155/2012/582453

[16] S. S. Moosavi, A. Djerdir, Y. Ait-Amirat, and D. A. Khaburi, "ANN based fault diagnosis of permanent magnet synchronous motor under stator winding shorted turn," Electric Power Systems Research, vol. 125, pp. 67-82, 2015. https://doi.org/10.1016/j.epsr.2015.03.024

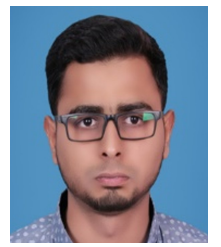

Ali Rohan received his B.S. degree in Electrical Engineering from The University of Faisalabad, Pakistan in 2012. From 2012 to 2013, he worked as a Development Engineer at Niagara group of Industries, Pakistan. From 2013 to 2015, he worked as a Project Engineer for Circle Club, Pakistan. From 2015 to 2016, he worked as a Project Manager for Steam Masters, Pakistan and also as a Lecturer at the Department of Electrical and Telecommunication Engineering, Government College University Faisalabad, Pakistan. In
2018, completed his M.S. degree in Electrical, Electronics and Control Engineering from Kunsan National University, Korea. Currently working as a Research Associate and pursuing his $\mathrm{Ph} . \mathrm{D}$. degree in Electrical, Electronics and Control Engineering from Kunsan National University, Korea. His research interests includes machine learning, AI, UAV's, power electronics, fuzzy logic, EV system, flywheel energy storage system.

E-mail: ali_rohan2003@hotmail.com

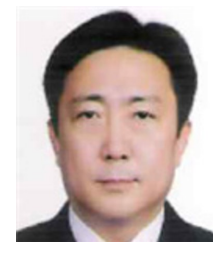

Sung-Ho Kim received his B.S. degree in Electrical Engineering from Korea University in 1984. He completed his M.S. and Ph.D. degrees in electrical engineering from Korea University in 1986 and 1991, respectively. In 1996, he completed his Post-Doc from Japan Hiroshima University. Currently, he is a professor at Kunsan National University. His research interests includes fuzzy logic, sensor networks, neural networks, intelligent control system, renewable energy system, fault diagnosis system.

E-mail: shkim@kunsan.ac.kr 